\title{
Improving Quality in a Cytopathology Laboratory
}

\author{
Zuhair M Mohammedsaleh ${ }^{1}$ \\ ${ }^{1}$ Department of Medical Laboratory Technology, Faculty of Applied Medical Sciences, University of Tabuk, \\ Kingdom of Saudi Arabia \\ Correspondence: Zuhair M Mohammedsaleh, Department of Medical Laboratory Technology, University of \\ Tabuk, Kingdom of Saudi Arabia. E-mail: zuhair.saleh966@gmail.com
}

Received: April 21, 2020 Accepted: August 12, 2020 Online Published: October 30, 2020

doi:10.5539/gjhs.v12n12p121

URL: https://doi.org/10.5539/gjhs.v12n12p121

\begin{abstract}
Quality assurance in a cytopathology laboratory is achieved by involving all the parties that contributes to cytopathology procedures. Quality assurance measures start with the laboratory directors to the cytopathotechnologists at work. High-quality results are achieved when all the parties work together by following the Standard operation procedures failure to which quality is undermined. Laboratory directors in a cytopathology laboratory are responsible for risk analysis and management. Proper risk analysis techniques help the lab manager to identify the weak points among the technologists. Proper management involves giving out good guidelines or instructions on what should be done to solve the problems that have been identified. There is the need to accredit and comply with the international accepted policies and procedures and excellent documentation to help deal with malpractices. Modern health care has faced a revolution due to a variety of factors, some of which are: newest techniques in laboratory medicine, trained staff operating diagnostic medical laboratories and ultra-modern analytical equipment. A Quality Management System (QMS) has been suggested by the ISO 15189 International Standard, which if followed, can help sustain and improve the testing services offered by diagnostic laboratories, such as cytopathology laboratories. Cytopathology laboratories should only employ laboratory technologists have undergone through the recommended training and have licenses from the regulatory bodies. The staff members of cytopathology laboratory should follow the standard operating procedures. SOPs help in coming up with high-quality results.
\end{abstract}

Keywords: cytopathology laboratory, health care, Quality Management System

\section{Introduction}

Medical cytopathology has completely changed its structure by the help of new and improved techniques involving Sciences and Technology. Liquid based cytology (LBC) has made obsolete the technique of diagnosing cancer simply on the basis of observing abnormal cells on smeared slides (Longatto Filho et al., 2005). Modern cytopathology laboratories operated by skilled staff has come into being after conventional cytology laboratories were upgraded using modern techniques of laboratory medicine and diagnostics. These laboratories use outcomes derived from different fields such as conventional cytology, molecular biology, analytical chemistry etc. to provide and piece together a workflow that can be used in testing and diagnostic services (Joste \& Gober-Wilcox, 2013). The gravity of the need of a trustworthy and reliable laboratory service can be judged by the clinical importance of the diagnostic assessments that the cytopathology laboratories carry out.

For the maintenance and stability of the rate of progress of a laboratory, certain traits should be stressed upon which include quality monitoring, patients' rights, standard operation procedures and standards of health care quality, which have been stated by modern cytopathology laboratories (Zima, 2010). An acceptable way of making sure that the calibre of testing procedures is sustained and the consistency of a diagnostic laboratory is improved is by working on assessment techniques and by structuring and stressing upon a Quality system and earning an official accreditation status (Archondakis, Vavoulis, \& Nasioutziki, 2016). A certified state agency or body is responsible for executing an accreditation process properly that works by performing a thorough laboratory assessment procedure, which makes sure that the said laboratory works according to the rules that are set and expected according to the types and extents of proceedings happening at the laboratory. Accreditation, in simpler terms, is the activity through which doctors or certified health professionals embark upon a clinical decision (Guzel \& Guner, 2009). 
Cytopathology has achieved a lot of milestones during the previous few decades. Cytopathology has changed faces over the years due to breakthroughs made in modern sciences and technology. The prevailing cytology approaches have upgraded to latest cytology approaches, some of which are: liquid based cytology, thin layer slide preparations and HPV DNA testing with the help of continuously evolving fields of scientific expertise, such as analytical chemistry, molecular biology, computer algorithm, and software programming. Patients seeking modern health care services now need to have compulsory access to Laboratory medicine and diagnostics. A health care system which expects to provide traits such as: maximum results, enhanced performance, efficiency and accuracy should make laboratories offering diagnostics an integral part of their system. A growing percentage of patients have started relying more and more on the diagnostic services offered by these laboratories which help make around $70 \%$ of the decisions by doctors and healthcare personnel (Hallworth, 2011; Hallworth et al., 2015). Cytopathology laboratories are well equipped to offer outstanding healthcare testing services, such as HPV DNA testing for cervical cancer screening, to provide reproducible and credible medical results (Vavoulidis et al., 2020). Obviously, due to the rising burdens of daily operations, clinical care and regulatory compliance in atomic pathology, the cytology community must be at the forefront of ensuring quality control and quality assurance (Heher, Chen, \& VanderLaan, 2017).

Cytopathology as a diagnostic technique examines cells of the body to determine the nature and cause of disease. Pap test was the first cytology test to be developed and has been used for over half a century for screening cervical cancer. Pap smear has been successful in almost all parts of the world. The practice of cytopathology started with a sample from the cervical region only, but now it has been expanded to include samples from other body sites. Cytopathology techniques such as Pap smear have been of much importance in preventing cancer. Population-based screening is now practiced in most of the developed countries (Davey et al., 2013). Therefore, it is important to implement quality control programs regarding the cytopathology procedures and steps. Quality is crucial when carrying out cytopathology procedures. Cytopathologists are required to follow the Standard Operating Procedures set by the WHO or their laboratory management. A lot of improvement has been achieved in the medical field of cytopathology due to introduction of top tiered laboratory diagnostic methods. Techniques of laboratory medicine in modern cytopathology have completely changed its course from the days when cancer screening was done on the basis of detecting abnormal cells on smeared slides. In present times, gold standards have been set with the advent of liquid-based Papanicolaou testing and various molecular biology techniques resembling the techniques that are used for DNA and RNA identification of Human Papillomaviruses from cervical and/ or histological samples along with computer-assisted screening. These new techniques have pushed the prevailing day Papinacolaou testing into a more modern one (Hidalgo et al., 2005; Ronco et al., 2007).

This Article evaluates and discusses ways of improving quality in a cytopathology laboratory.

\section{Quality Control (QC)}

Quality is an expression that can be used to describe the standard of health care and medical services offered. It is set as a threshold of a calibre, which if not reached, can lead to rejection of the services and final results offered to the patients to assist with their necessities. That is why, according to laboratory diagnostic medicine, quality is defined as distinctive traits that a diagnostic service or procedure should be offering to its patients to complete their necessities and in return also provide satiety to the clinicians that their demands have been met (Burnett,1999; Branca \& Longatto-Filho, 2015)

One of the functions of a modern diagnostic laboratory should be to offer excellent assistance to its patients and in addition, also serve to improve and keep consistent the standards that it has introduced. Apart from that, once an investigation has been carried out to grade the quality of a laboratory, the laboratory should make sure to take all necessary actions and precautions to keep consistent the standard of its services and the expectations it has set to the patients. These goals can be obtained by a diagnostic laboratory once it takes into effect an organized Quality Management System (QMS) that is composed of Quality Control and Assurance System (QCAS). QC gives an idea of the excellence of the services provided, and gives confirmation if it fulfils the criteria of excellence it has set, while QA grades whether they achieve their set goals or not (Archondakis, 2015).

Quality control is the practice of ensuring that both accuracy and precision are maintained when processing the cytopathology samples. Quality control samples and reagents should be of high integrity as well as meet the necessities of proficiency testing. In a cytopathology laboratory, quality control helps in identifying the mistakes with the samples and calibration of machines (Eden, 2015). The practice of quality control ensures all the laboratory tests are done in the right way hence producing accurate results. QC samples should look similar to patient samples. Usually, it is necessary to repeat quality control testing to certify precision and accuracy of the patient's results (Eden, 2015). Precision is the level of concurrence amongst repetitive measurements of the 
identical characteristic on an equivalent sample. On the other hand, accuracy is checked whether the end results are close to what is expected at the end of the procedure.

\section{ISO 15189 and Medical Laboratories}

ISO 15189 gives in details how a medical laboratory is supposed to work or operate so as to produce quality results. ISO 15189 helps the medical laboratories to come up with the appropriate management system and assessing their competence. In many countries, the accreditation bodies use ISO15189 criteria to confirm and recognize the laboratory's competence. ISO 15189 stress that the laboratory technologist, reagents, equipment among other factors must be competent (James et al., 2014). In the past few years, laboratory medicine has been strengthened into a tool that can be of resource for the present day healthcare system. This has been achieved by introducing new experimental techniques and top tiered analytical equipment. Quality is attribute that is given the most importance if actively managed by the personnel of each diagnostic laboratory. To help achieve the targets of Quality Management, some approaches can be set into motion by introducing QC and QA strategies respective to the requirements set by ISO 15189:2012 (Archondakis, 2015; Archondakis, Vavoulidis, \& Nasioutziki, 2016).

ISO 15189 has been advanced with extra prerequisites in its most recent release. These prerequisites are both specialized and administrative that can help modern diagnostic laboratories such as cytopathology ones to deliver top class quality testing services and outcomes to healthcare. An outlined and organized QCAS set as a component of a QMS is important for steady and proper checking of real-time quality standards that are an attribute of any specific laboratory examination in any time period. QC and QA approaches have been set for subjects of Laboratory medicine such as Biochemistry and haematology since the start, but they have been introduced to cytopathology just recently (Whitehead, Browning, \& Gregory, 1973; Bullock \& Wilde, 1985). Implementing QC and QA in cytopathology has always been a little complicated because of the reason that a cytopathology report can be affected by many practical reasons including variations in the working skills among cytopathologists and technical expertise among cytotechnologists. In addition, inaccuracy in diagnostics can be as a result of inadequate attention, exhaustion, and inadequate time while carrying out the analytical duties by the trained scientific staff; the errors can turn into manifold when they are over worked (Frable, 2007).

Only Laboratories that meet the ISO 15189 criteria should be accredited to ISO 15189. A cytopathology laboratory should meet the following criteria before for it to be ISO certified.

Quality assurance and control practices in a cytopathology lab include the use of Intra-laboratory and extra-departmental consultations, correlation of histopathology and cytological specimens and review of the diagnostic reports. The following are the quality assurance and control measures; Laboratory Directors: The Director of Cytopathology laboratory should be a well-qualified physician with a postgraduate degree in pathology including training and experience in cytopathology procedures.

\subsection{Cytotechnologists}

These are the staff members involved in the processing of the samples. This position requires a suitably qualified person, most probably one who possesses the first degree in medical laboratory sciences and a master in histo-cytopathology (Herbert et al., 2014). However, the qualification criteria may differ from one country to another.

\subsection{The Laboratory Facility}

The laboratory should meet the recommended standards. It must be well organized and ventilated. Biosafety cabinets and the fridges should be in good working condition. Chemicals should be kept away from direct sunlight or fire since this may destroy them (Pouliakis et al., 2014). Fridges help to maintain the recommended temperatures for some reagents. Only laboratory staff members should access the facility. Patients should wait for or receive the results at the reception.

\subsection{Safety Precautions}

The staff must be in protective gear when working. Infectious materials should be discarded safely. The laboratory should have fire extinguishers to put off the fire in case it arises. All laboratory technologists must be in lab coats and gloves when working (Panel, 2012). It is vital to post and test all the fire precautions.

\subsection{Equipment}

The director of lab services must ensure all the equipment are of high quality and are in proper conditions (Director-ihsm, 2016). Only qualified technicians should service the machine and ensure the malfunctions are fixed. Malfunctions affect the quality of results. All cytopathology laboratories should consider using the most modern equipment that produces high-quality results (Joste \& Gober-Wilcox, 2013). 


\subsection{Specimen Collection}

The person receiving the samples at the laboratory should ensure that the samples meet the recommended standards. The pathologists must collect the samples using the right procedures. The right procedure for specimen rejection should be applied. The rejected sample should be accompanied by a rejection report indicating why the samples were rejected. Only qualified medical practitioners should take samples from the patients (Sheaff \& Singh, 2013).

\subsection{Preparation, Fixation, and Staining Methods}

The staff should ensure that the samples have the patient's detail. The request form should have the requesting doctor's and patient's information. The slides in the laboratory should be assigned a number corresponding to the number of the patient's request form or a unique identifier. All specimens that do not meet the processing criteria should be rejected using the set standards for rejection. It is good to fix conventional cell samples while they are wet. Most of the cytological samples are processed using through Papanicolaou procedures (Sheaff \& Singh, 2013). The staining solutions and the reagents should be covered immediately after. Some of the solutions are flammable and therefore, immediate covering reduces risks (Shambayati, 2011). The laboratory reagents should both the manufacturing and the date of preparations and the expiry dates.

\subsection{Slide Evaluation and Workload}

Cytopathotechnologists should not over work. They should only handle the recommended number of slides per day. Samples from cases that require urgency should be given priority. A technologist should only work for eight hours in a day. Overworking is highly discouraged since it can cause poor service delivery (Branca \& Adhemar, 2015). The director of laboratory services is responsible for evaluating the number of slides or workload per staff. However, internationally set standards should be followed.

\subsection{Cytologic Terminology}

Bethesda is the recommended system for interpreting most of the cytology samples such as an ectocervical or endocervical sample. On the other hand, Nongynecologic materials should be interpreted using the appropriate medical terminologies (Pitman et al., 2014).

Laboratory records, logs and files: Proper record keeping is very important in the laboratory. The person receiving the samples at the laboratory should ensure that he/she has assigned them appropriate numbers depending on the laboratory's numbering systems. Laboratory records must be kept for a long period for future reference. The samples should not be kept for a period less than five years. Samples that need further research or investigation should be kept safely for a longer period (Pantanowitz \& Austin, 2014). The results should also be recorded well in the laboratory's record book before the results are dispatched to the client or the doctor.

\section{Risk Analysis and Management Analysis}

Risk is an expression that is widely used to define 'being susceptible to any danger, peril or threat and the mentioned catastrophic event being likely to happen in a set time span' (Alberg, Hatfield, \& Huxley, 1996). Evaluating or estimating risk can be achieved by assessing the likely jeopardizes that can happen as a result of an unforeseen and unfortunate occurrence. It is in actual a methodical report that functions to quantitatively reveal the results and impact of any immediate harm that might befall, keeping in mind the likely occurrence and incidence of the outcome. It is a method which includes gathering, arrangement and assessment of data and information obtained from various sources, considering the attainment of an end goal to evaluate the degree of severity and probability of a specific risk or danger and the examples of circumstances in which hazard related parameters may emerge (Kohn, Corrigan, \& Donaldson, 2000; Morgan, 2000). Any specific venture that is subject to any harm can investigate its risk possibilities and to achieve that result, medical diagnostic laboratories can pose as promising endeavours of that kind. One exceptional case of such a laboratory is the modern cytopathology laboratory that offers its patients distinctive assistance, such as game plans for examination requests and readiness of patients, accumulation, transportation, stockpiling, investigation and accurate assessment of clinical specimens, and in addition, translation, reporting and counselling is offered (Archondakis, 2015).

After cytophathotechnologist has identified a risk in the laboratory, it is important to carry out the quality control process. It is the role of the management to identify the severity and the risk the underlying problem posed to the laboratory staff. Therefore, the management can apply tools and come up with procedures that can put a value on the identified risk. In most cytopathology laboratories, the director gives the steps that should be followed to report the accidents and incidences however small they are (Pantanowitz \& Parwani, 2014). The recorded data is then recorded, and this enables the manager to evaluate the issue accordingly. 


\section{Risk Management}

There is the need to accredit and comply with the international accepted policies and procedures and excellent documentation to help deal with malpractices (Stavros \& Nasioutziki, 2016). Cervical cytology claims for cytology laboratories, pathologists, and cytotechnologist have been rapidly increasing over the years. Currently, the severity of the claims is very high thus putting pathologists at par with obstetricians and anesthesiologists (Kocjan et al., 2013). It is important to check the details of a cytological specimen whenever a patient claims that his or her results were misleading. All cytology laboratories in the United States are expected to be in full compliance with the States and the Federal laws to avoid malpractices. It is importance to make sure the entire laboratory follows the documented workload policy. In the case of malpractices, the laboratory director should establish whether the specimen underwent through the right procedure and if the results were recorded well. The Clinical Laboratory Improvement Amendments of 1988(CLIA) raised the following risk and quality, management concerns; proper record keeping of all the cytologic specimens, proper slide handling, and retention, amended reports from retrospective review and workload limits.

\section{Equivalent Knowledge and Skills}

Medical technologists in cytopathology laboratory should have enough knowledge on all cytology procedures and be in a position to follow them from the first step to the last without any difficulties. The technologist must understand and carry out their duties with minimum supervision (Crothers et al., 2013). Knowledge must be professionally acquired through training at a medical school. The technologist must have enough knowledge of basic principles, concepts, and cytopathology in addition to laboratory mathematics and statistics and skills in carrying out all the specialized tests (Crothers et al., 2013). Knowledge of mathematics and statistics is vital in establishing quality controls, calculate and correlate test results and troubleshoot procedures and equipment. The cytopathotechnologists should also have enough knowledge of related disciplines such as physiology, anatomy, epidemiology among others. The knowledge and understanding of the medicolegal requirements, internationally recognized standards and requirements of the accrediting agency is vital (Crothers et al., 2013).

Supervisory controls: Each cytopathologist should have supervisory control skills. This skill enables a technologist to give instructions, give priority to the urgent results, set appropriate deadlines and define objectives. However, the overall supervision is done by the director of laboratory services in the cytopathology laboratory. A supervisor has the following roles and duties in the laboratory; assigns duties by giving instructions which cover the steps to be followed. Cytopathologists fresh from college should work under a supervisor who gives the instructions and oversees the work done. The supervisor monitors the progress of all the laboratory procedures (Crothers et al., 2013). The supervisor in a cytopathology laboratory indicates what is to be done, quality of work expected, problems anticipated, deadlines, the priority of assignments and suggests ways of handling and solving problems that may arise. The laboratory supervisor should closely control the work by reviewing the progress and on completion the accuracy of results, and adherence to instructions and established procedures. The director ensures that the results and cytopathology methods followed are technically accurate and in accordance with established procedures. The supervisor should assist the new technologist to learn all the test methods.

Health advocate: Cytopathologists should responsibly use their knowledge gained in medical school and their influence to improve the well-being of patients and the entire population they serve (Yu, 2011). They should demonstrate awareness of the risk factors that contribute to a disease, the main reasons for screening the community against a particular disease condition and the role of other healthcare professionals in such processes (Smart et al., 2016). The cytopathologist should demonstrate knowledge of all the factors that contribute to a disease within a given community, the importance of screening and the role of technologists in this process. Cytopathologists should also work towards promoting the health of all people. They should demonstrate safe practices when carrying out the procedures on patients and when processing the specimens in the laboratory (Mourtzikou et al., 2013). Scholars; Cytopathologists should always be ready to advance their knowledge by leading wide, attending cytopathology workshops and seminars, and participate in assessment programs.

Rigid measures and effective systems must be connected keeping in mind the end goal to keep up or even enhance the nature of the research facility laboratory performance and the relating indicative assessments that are completed. Present-day Cytopathology laboratories need to make a perfect mix of internal QC and outside QA systems that will cover their wide testing portfolio, guaranteeing amazing guidelines for all the performed examinations, both morphological and molecular. M-Health technology plays an evident role in improving the laboratory QCAS and the examination level. Introducing Computer algorithms and evolutionary user interfaces has catalysed the analytical examinations and managing examination requests by immediately giving rise to examination lists. 


\section{Way to Evaluate Laboratory Performance}

The way to evaluate laboratory performance is by carrying out internal quality control on a routine basis. Positive and negative controls help to know whether the test was performed well without omitting any step. Positive controls monitor the calibration of the system thus providing the assurance that the right procedures are followed (Allen et al., 2013). Cytopathologists should run positive control besides the tests every day. Negative controls evaluate the specificity of the tests to identify false-positive staining results. They include negative tissue controls. Cytopathologist should select the best tissue controls. At the end of the staining or processing, the pathologists compare the three slides, that is, the sample slide, the negative control slide, and the positive control slide to know whether the cytopathologists carried out the staining procedure well (Denton et al., 2010). External quality control is also an important practice of ensuring that the laboratory is performing as per the international set standards. The laboratory manager should ensure that sample slides are exchanged with slides from other laboratories to assess the quality of the testing procedure and the results obtained (Ázara et al., 2013).

\section{Standard Operation Procedures (SOPS)}

The main specific role to improve quality in the laboratory is making sure that all the staff members strictly follow the standard operation procedures (SOPS). The standard operating procedures cover the following areas in the laboratory; quality control, routines, performance management, and replication and growth. Routines; in a cytopathology laboratory, the SOPs helps the technologists to carry out their duties with much ease. SOPs should be well documented in a flow chart and placed on the walls or where, or the staff members can read see or reach them easily (Bibbo \& Wilbur, 2014). SOPs makes the laboratory procedures easy to follow either in the print out or on the computer.

Quality control; SOPS in a cytopathology laboratory help to minimize errors or variations that occur when the technologist are handling many samples at a time. Each laboratory should have SOPs for quality (Maruta et al., 2012). SOPs help in the director of laboratory services and the senior technologist to discard results that fail to meet quality and request for a repeat of the whole procedure. Performance management; SOPs in a cytopathology laboratory helps to make it easier to check the technologist's performance appraisals (International Organization for Standardization, 2012). The director of laboratory services should write each employee's job description and give them target level for each SOP in the job description. The staff's SOPs should just have the procedures most crucial to the job (Clary et al., 2013). Replication and Growth: SOPs helps to replicate processes across the Laboratory if the health institution has more than one laboratory in different areas. This aid in making sure that the end results are of high quality. Usually, it is easy to compare the performance of the cytopathotechnologists who perform the same SOP as part of their jobs.

\section{Factors Influencing Development and Training of Cytopathology Staffs}

Automation; cytopathotechnologists in the United Kingdom are under pressure to deliver quality results by decreasing the turnaround time while at the same time producing quality results. Technology changes from time to time, and therefore the State is faced with a challenge of sending cytotechnologists for training whenever the new machine that proves to be of high quality is invented (Oltvai, 2012). The government incurs much cost in this. In addition, the aged cytopathotechnologists who trained many years ago might not be willing to learn the modern technologies or they are not in a position to learn the new technology.

Training period; it requires at least two years training a cytopathotechnologist. Not many Medical officers or laboratory technologist are willing to undergo another two years of training after attaining the first degree. Most of the doctors and medical laboratory technologist fail to advance and opt to work as general practitioners. However, it is not possible to reduce the training period because many units and practical lessons have to be covered. The hospital attachments programs are also time consuming. Most of the technologists feel demoralized since they work for free during the internship period. Therefore, many students for health science carries that are not time demanding.

To improve performance; this is another factor that influences the training of cytopathology staff. Short courses help in updating the technologists the changes or new technologies in place. In addition, the short courses assist the staff members to refresh on the skills they acquired in college (Khurana, 2012). Cytopathology staffs who attend seminars and short courses are likely to produce quality results since they keep on updating themselves.

Shortage of cytopathologists; many countries across the world are faced with the shortage of cytopathologists. There is the need to encourage more doctors to major in this carrier since there is demand for more cytopathologists due to the increase in the number of cancer-related cases in most parts of the world. However, the cost and time required to train cytopathologist discourage most of the doctors from enrolling from cytopathology 
career. The government should consider offering partial or full scholarships to doctors willing to pursue a cytopathology career.

\section{Conclusion}

From the above discussion, one can conclude that it is vital for all cytopathology laboratories to ensure that they produce high-quality results. High-quality results are achievable strict adherence to the standard operating procedures, employing qualified and competent staff members, team-work and adopting the most modern testing technologies. The cytopathotechnologists should also undergo specialized training aimed at equipping them with all the test procedures that take place in a cytopathology laboratory. The director of the laboratory services should ensure that all the tests underwent through the right procedure. Proper supervision ensures that the results are of high quality. New technologies like automation reduce the time taken to produce results and improve quality. The cytopathology community needs to improve the quality in this type of laboratory. Some strategies for improvement include the introduction of an electronic patient data bank, as opposed to a manual one, to enable pathologists to access all information from other laboratories that might have been omitted in a clinician's notes.consistent learning, professionalism, reduction in batching, electronic medical records, High-quality patient care, productivity, quality and safety, competent staff and proactive managerial leadership are just some of the key elements to success and improvement in cytopathology laboratories.

Health can help shape the administration and have a direct effect on the QMS and its accreditation requirements. The slow and steady implementation of m-Health applications will change the course of the way cytopathology laboratories will develop, implement and keep in check the QC and QA strategies in the subsequent years.

\section{Competing Interests Statement}

The author declares that there are no competing or potential conflicts of interest.

\section{References}

Allen, D. C. (2000). Histopathology Reporting: Guidelines for surgical cancer and Gynecology Clinics of North America, 40(2), 199-210. https://doi.org/10.1016/j.ogc.2013.02.001

Ázara, C. Z. S., Manrique, E. J. C., Alves de Souza, N. L., Rodrigues, A. R., Tavares, S., \& Amaral, R. G. (2013). External quality control of cervical cytopathology: interlaboratory variability. Acta cytologica, 57(6), 585-590. https://doi.org/10.1159/000353843

Bibbo, M., \& Wilbur, D. (2014). Comprehensive cytopathology. Elsevier Health Sciences.

Branca, Margherita, Adhemar, \& Longatto-Filho. (2015) Recommendations on Quality Control and Quality Assurance in Cervical Cytology. Acta cytologica， 59.5(2015). 361-369. https://doi.org/10.1159/000441515

Clary, K. M., Davey, D. D., Naryshkin, S., Austin, R. M., Thomas, N., Chmara, B. A., ... \& Tworek, J. (2013). The role of monitoring interpretive rates, concordance between cytotechnologist and pathologist interpretations before sign-out, and turnaround time in gynecologic cytology quality assurance: findings from the College of American Pathologists Gynecologic Cytopathology Quality Consensus Conference Working Group 1. Archives of pathology \& laboratory medicine, 137(2), 164-174. https://doi.org/10.5858/arpa.2012-0120-CC

Crothers, B. A., Jones, B. A., Cahill, L. A., Moriarty, A. T., Mody, D. R., Tench, W. D., \& Souers, R. J. (2013). Quality improvement opportunities in gynecologic cytologic-histologic correlations: findings from the College of American Pathologists Gynecologic Cytopathology Quality Consensus Conference working group 4. Archives of pathology \& laboratory medicine, 137(2), 199-213. https://doi.org/10.5858/arpa.2012-0250-OA

Davey, D. D., Goulart, R., \& Nayar, R. (2013). Cytopathology Education and Technology Consortium. 2013 statement on human papillomavirus DNA test utilization. Cancer Cytopathol. https://doi.org/10.1002/dc.23094

Denton, K. J., Bergeron, C., Klement, P., Trunk, M. J., Keller, T., \& Ridder, R. (2010). The sensitivity and specificity of p16INK4a cytology vs. HPV testing for detecting high-grade cervical disease in the triage of ASC-US and LSIL Pap cytology results. American Journal of Clinical Pathology, 134(1), 12-21. https://doi.org/10.1309/AJCP3CD9YKYFJDQL

DIRECTOR-IHSM. (2016). Retrieved 20 July 2016, from http://L DIRECTOR-IHSM-hsmdghs-bd.org

Eden, M. (2015). Quality Control In Clinical Laboratory Samples MLO. Retrieved from Mlo-online.com

Elsheikh, T. M., Austin, R. M., Chhieng, D. F., Miller, F. S., Moriarty, A. T., \& Renshaw, A. A. (2013). American 
Society of Cytopathology workload recommendations for automated Pap test screening: developed by the Productivity and Quality Assurance in the Era of Automated Screening Task Force. Diagnostic cytopathology, 41(2), 174-178. Geneva, Switzerland: ISO. https://doi.org/10.1002/dc.22817

Heher, Y. K., Chen, Y., \& VanderLaan, P. A. (2017). Measuring and assuring quality performance in cytology: a toolkit. Cancer cytopathology, 125(S6), 502-507. https://doi.org/10.1002/cncy.21831

Herbert, A., Anic, V., Cochand - Priollet, B., Dina, R., Ehya, H., Eide, M. L., ... \& Oliveira, M. H. (2014). Training and practice of cytotechnologists: a discussion forum focused on Europe. Cytopathology, 25(5), 307-315. https://doi.org/10.1111/cyt.12201

International Organization for Standardization (ISO). (2012). ISO 15189: Medical Laboratories Requirements

James, D., Ames, D., Lopez, B., Still, R., Simpson, W., \& Twomey, P. (2014). External quality assessment: best practice. Journal of clinical pathology, jclinpath-2013. https://doi.org/10.1136/jclinpath-2013-201621

Joste, N., \& Gober-Wilcox, J. (2013). The Modern Cytology Laboratory: Moving Beyond the Pap Test. Obstetrics. https://doi.org/10.1016/j.ogc.2013.02.001

Khurana, K. K. (2012). Telecytology and its evolving role in cytopathology. Diagnostic cytopathology, 40(6), 498-502. https://doi.org/10.1002/dc.22822

Kocjan, G., Gray, W., Levine, T., Kardum-Skelin, I., \& Vielh, P. (2013). Diagnostic cytopathology essentials.

Maruta, T., Motebang, D., Mathabo, L., Rotz, P. J., Wanyoike, J., \& Peter, T. (2012). Impact of mentorship on WHO-AFRO strengthening laboratory quality improvement process towards accreditation (SLIPTA). African Journal of Laboratory Medicine, 1(1), 8. https://doi.org/10.4102/ajlm.v1i1.6

Mourtzikou, A., Stamouli, M., \& Athanasiadi, E. (2013). Improvement of Clinical Laboratory Services through Quality. International Journal of Reliable and Quality E-Healthcare (IJRQEH), 2(2), 38-46. https://doi.org/10.4018/ijrqeh.2013040103

Oltvai, Z. (2012). Conceptual Advances in Pathology. London: Elsevier Health Sciences

Panel, B. B. R. (2012). Guidelines for safe work practices in human and animal medical diagnostic laboratories. Morbidity and Mortality Weekly Report, 61.

Pantanowitz, L., \& Parwani, A. V. (2014). Practical Informatics for Cytopathology. New York, NY: Springer New York. https://doi.org/10.1007/978-1-4614-9581-9

Park, S., Pantanowitz, L., Parwani, A. V., Wells, A., \& Oltvai, Z. N. (2012). Workflow organization in pathology. Clinics in laboratory medicine, 32(4), 601-622. https://doi.org/10.1016/j.cll.2012.07.008

Pitman, M. B., Centeno, B. A., Ali, S. Z., Genevay, M., Stelow, E., Mino-Kenudson, M., ... \& Layfield, L. J. (2014) Standardized terminology and nomenclature for pancreatobiliary cytology: the Papanicolaou Society of Cytopathology guidelines. CytoJournal, 11(2), 3. https://doi.org/10.4103/1742-6413.133343

Pouliakis, A., Margari, N., Spathis, A., Kottaridi, C., Stamouli, M., Mourtzikou, A., ... \& Karakitsos, P. (2014). ISO 15189: 2012 Technical Requirements for Cytopathology Laboratory Information Systems. International Journal of Reliable and Quality E-Healthcare (IJRQEH), 3(3), 58-80. https://doi.org/10.4018/ijrqeh.2014070104

Shambayati, B. (2011). Cytopathology. Oxford: Oxford Univ. Press.

Sheaff, M. T., \& Singh, N. (2013). Cytopathology: An introduction. London: Springer https://doi.org/10.1007/978-1-4471-2419-1

Smart, L. M., Buchan, M., Cropper, A. J., Cross, P. A., Denton, K. J., Fraser, M. A., \& Wilson, A. (2016). BAC recommended code of practice for cytology laboratories participating in the UK cervical screening programmes 2015: a secondary publication. Cytopathology, 27(1), 8-34. https://doi.org/10.1111/cyt.12320

Stavros, A., Vavoulidis, E., \& Nasioutziki, M. (2016). The Use of Mobile Health Applications for Quality Control and Accreditational Purposes in a Cytopathology Laboratory. M-Health Innovations for Patient-Centered Care, 262. https://doi.org/10.4018/978-1-4666-9861-1.ch013

Tötsch, M., Cuvelier, C., Vass, L., \& Fassina, A. (2012). The UEMS section/board of pathology, chapter 6: requirement for recognition of postgraduate training in pathology: a presentation of the paris document. Cytopathology, 23(5), 295-299. https://doi.org/10.1111/cyt.12020

Vavoulidis, E., Nasioutziki, M., Mareti, E., Karpa, V., Tsabazis, N., Pratilas, G. C., ... \& Dinas, K. (2020). Quality 
Control and Assurance in ISO15189: 2012 Molecular Cytopathology Laboratories for HPV DNA Testing. Acta Scientific Microbiology, $\quad 3$, 06-12. https://doi.org/10.31080/ASMI.2020.03.quality-control-and-assurance-in-iso151892012-molecular-cytopath ology-laboratories-for-hpv-dna-testing

Wilson, A. (2015). The role of Cytotechnologists in quality assurance and audit in non - gynaecological cytology. Cytopathology, 26(2), 75-78. https://doi.org/10.1111/cyt.12246

Yu, G. H. (2011). Goals and guidelines for residency training in cytopathology. Diagnostic cytopathology, 39(6), 455-460. https://doi.org/10.1002/dc.21436

\section{Copyrights}

Copyright for this article is retained by the author(s), with first publication rights granted to the journal.

This is an open-access article distributed under the terms and conditions of the Creative Commons Attribution license (http://creativecommons.org/licenses/by/4.0/). 